\title{
Light: Duality or Discontinuity?
}

\author{
Wei-Xing Xu \\ Newtech Monitoring Inc., Oshawa, Canada \\ Email: dumplingcat_2@yahoo.com
}

Received January 20, 2013; revised February 21, 2013; accepted February 28, 2013

\begin{abstract}
Based on the perturbation theory of quantum mechanics, we found that the time for a system to absorb a photon is the period of the light wave. This result gives us an exact definition of a photon, that is, a photon is just a single period of light wave. Furthermore, the detail analysis of the emission and absorption of the light wave in a system tells us that the light wave is not continuous as we believed before but discontinuous instead. Based on this result, we elucidate the origin of the particle-wave duality of the light/electromagnetic wave.
\end{abstract}

Keywords: Duality; Photon Dimension; Spectrum Line Width; Light Absorption/Emission

\section{Introduction}

Since the beginning of science, the human being already knew the importance of the light in their life. They paid much attention to understanding the property of light, such as how the light propagates and what is composed of the light. First, they imagine the light as the wave-like phenomenon and later on, this wave-like hypothesis get the experimental support, for example, double slit experiment [1]. Among the wave-like supporters, the famous ones are Aristotle, Christian Huygens, Thomas Young, James Clerk Maxwell. It seems the wave-like theory of light is generally accepted in scientific fields. On another side, the particle-like hypothesis of the light is also proposed at the beginning of science. With the more and more experimental support for the wave-like theory, the voice of the particle-like hypothesis becomes weaker and weaker but never stops. Finally, the experimental evidence of the particle-like hypothesis of light comes, such as photoelectric effect $[2,3]$, Compton effect. Among the supporters of the particle-like theory of light, the famous ones are Democritus, Isaac Newton, Max Planck, Albert Einstein, Arthor Compton. Both sides all have strong experimental evidence, no one can beat another completely. The situation continues until the birth of quantum theory [4].

The quantum theory shows us the nature sometime shows the wave-like behavior but sometime shows the particle-like behavior. Max Planck, Louis de Broglie, Niels Bohr and a long list of famous scientist make great contribution to the quantum mechanics development. Finally, we just take the explanation of the wave-particle duality as it is and think the wave-particle duality is the basic property of the nature. Nobody give the detail about how the two totally different property, wave and particle, are unified in the nature [5-7].

In this paper, we start with the perturbation theory of quantum mechanics and hope to get more reasonable explanation about the duality of the light/electromagnetic wave.

\section{Theory}

Based on the time-dependent theory, the total Hamiltonian is of the form:

$$
H(r, t)=H_{0}(r)+\lambda H^{\prime}(r, t)
$$

where $H_{0}(r)$ is the Hamiltonian of the unperturbed system; $H^{\prime}(r, t)$ is the perturbation applied onto the system at $t=0 ; \lambda$ is a parameter of smallness.

Following the conventional procedure, we can get,

$$
C_{k}(t)=\frac{H_{k l}^{\prime}(r)}{\mathrm{i} \hbar} \int_{-\infty}^{t} \mathrm{e}^{\mathrm{i} \omega_{k l} t^{\prime}} f\left(t^{\prime}\right) \mathrm{d} t^{\prime}
$$

where $C_{k}(t)$ is the coefficient of the wave function under perturbation at $t . \quad C_{k}^{2}(t)$ describes the probability of the system transition from $\Psi_{1}$ to $\Psi_{k}$ at $t$.

The Equation (2) is familiar to those who take the advanced quantum mechanics at their graduate study. Here starting with this general equation, we hope to elucidate several basic questions about the interaction between the light and matter.

\subsection{How Much Time for a System to Absorb a Photon?}

In fact, this question is equivalent to what the space dimensions of a photon? In order to simplify the discussion, 
we take the system only with two states as an example (see Figure 1) and under the dipole moment approximation, the perturbation terms takes the form,

$$
H^{\prime}(r, t)=-\mu_{x} E=-\mu_{x} E_{0} \cos (2 \pi \omega t) .
$$

Then, the Equation (2) can be simplified as

$$
C_{2}(t)=\mu_{x}^{21} \frac{E_{0}}{2}\left[\frac{1-\mathrm{e}^{\frac{\mathrm{i}}{\hbar}\left(E_{2}-E_{1}+\hbar \omega\right) t}}{E_{2}-E_{1}+\hbar \omega}+\frac{1-\mathrm{e}^{-\frac{\mathrm{i}}{\hbar}\left(E_{2}-E_{1}-\hbar \omega\right) t}}{E_{2}-E_{1}-\hbar \omega}\right]
$$

where $\mu_{x}^{21}$ is the transition dipole moment defined by

$$
\mu_{x}^{21}=\int \Psi_{2}^{*}(x) \mu_{x} \Psi_{1}(x) \mathrm{d} x
$$

The first term in Equation (4) describes the emission of photon from the system; the second one corresponds to the absorption of photon by the system.

Now we only consider the absorption of photon by the system. Then we find, when $E_{2}-E_{1} \rightarrow \hbar \omega$, the second term becomes $0 / 0$. Based on L'Hopital rule,

$$
\begin{aligned}
& \lim \left[\frac{1-\mathrm{e}^{-\frac{\mathrm{i}}{\hbar}\left(E_{2}-E_{1}-\hbar \omega\right) t}}{E_{2}-E_{1}-\hbar \omega}\right]_{E_{2}-E_{1}-\hbar \omega \rightarrow 0} \\
= & \frac{\mathrm{i} t}{\hbar}\left[\mathrm{e}^{-\frac{\mathrm{i}}{\hbar}\left(E_{2}-E_{1}-\hbar \omega\right) t}\right]_{E_{2}-E_{1}-\hbar \omega \rightarrow 0}=\frac{\mathrm{i} t}{\hbar} .
\end{aligned}
$$

So, the Equation (4) becomes

$$
C_{2}(t)=\mu_{x}^{21} \frac{E_{0}}{2} \frac{\mathrm{i} t}{\hbar} .
$$

In fact, $\mu_{x}^{21} \frac{E_{0}}{2}$ can be selected as $\hbar \omega_{21}$, but doesn't lose generality, therefore,

$$
C_{2}(t)=\hbar \omega_{21} \frac{\mathrm{i} t}{\hbar}=\mathrm{i} \omega_{21} t .
$$

We know $C_{2}^{2}(t)$ corresponds to the probability of the transition from state 1 to state 2 . After the system absorbs a photon and be excited to the state $2, C_{2}^{2}(t)$ should be 1, so,

$$
C_{2}^{2}(t)=\left(\mathrm{i} \omega_{21} t\right)\left(\mathrm{i} \omega_{21} t\right)^{*}=1
$$

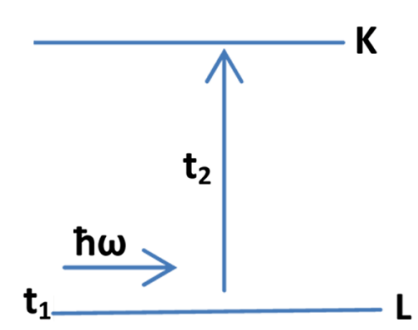

Figure 1. The illustration for a two levels system in the process of emission and absorption of a photon.
We obtain,

$$
\omega_{21} t=1 .
$$

This result tells that the time for the system to absorb a photon is the period of the light wave and therefore, the space dimension of a photon in its propagation direction is just one complete period or wavelength, therefore, we can call each single period of light wave as a photon. For the different frequency of light/electromagnetic wave, the spacetime dimension for a photon is different, the higher the frequency, the smaller the spacetime of the photon. In fact we can design an experiment to verify the time for a system to absorb a photon is the period of the light. We can put a switch between the light source and system, if the switch turn-on and turn-off time is less than the period of the light wave, then, the system cannot absorb the light. At moment, we just don't know whether this kind of switch exists or not. If yes, then to verify the conclusion here is much easier. Our result here also reveals that the time for the system to absorb a photon depends on the frequency of light and determines the line width of the absorption spectrum. That means for the absorption spectrum, the nature line width is $2 T$, where $T$ is the period of corresponding light/electromagnetic wave.

\subsection{Light/Electromagnetic Wave Is Continuous or Discontinuous?}

To my knowledge, the light/electromagnetic wave is believed to be continuous wave like water wave, but sometime behaviors like a particle. Nobody tells us why and how. Therefore, we call this property of light/electromagnetic wave as duality.

We still take two levels system as an example, First, the system absorbs a photon from its surroundings and be excited from $L$ level to $K$ level, then, gives up a photon to its surroundings and systettm decay from $K$ level to $L$ level. So, if we take this two levels system as a source of light, then, the light cannot be continuous but like dashed line instead (See Figure 2). The minimum gap between two photons is $t_{g}=t_{1}+t_{2}+t_{3}+t_{4}$.

From the discussion above, we know $t_{2}=t_{4}=T$, but normally, $t_{1} \neq t_{3}$, therefore, $t_{g} \geq 2 T+t_{1}+t_{3}$. From the literature [4], we know that the Einstein coefficient $A$

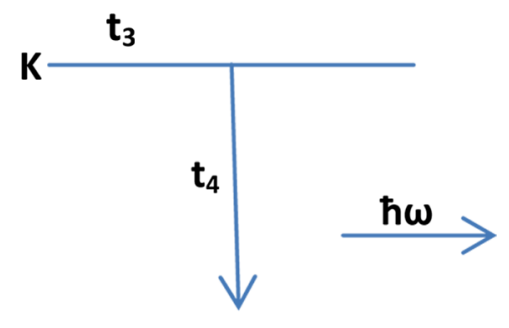



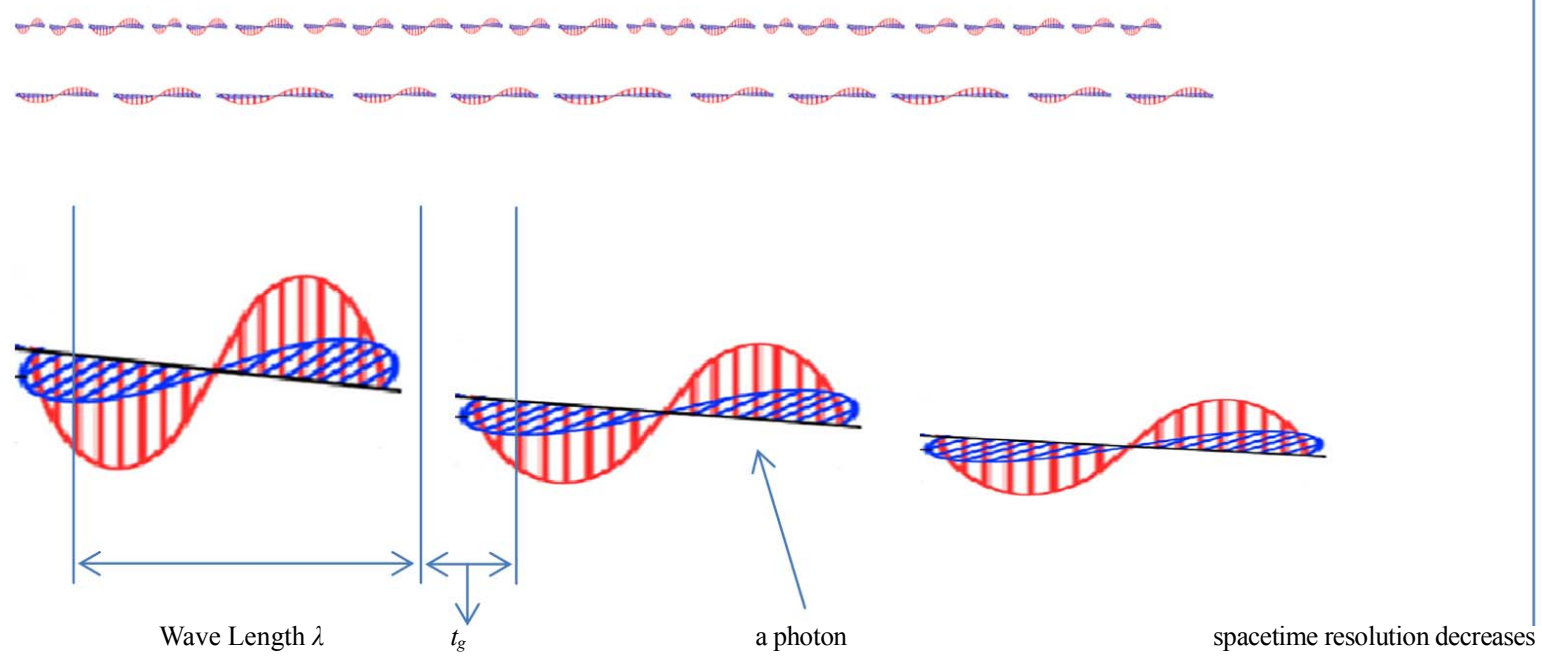

Figure 2. The illustration of the light/electromagnetic wave.

represents the decay rate per unit time per atom (here we don't consider the stimulated emission). For two levels system, the $1 / A$ represents the lifetime of the excited state and correspondingly, the Einstein coefficient $B$ represents the probability rate of the system excited from $L$ level to $K$ level (see Figure 1) per unit time per atom. Therefore, the $1 / B$ represents the lifetime of the system at the ground state under the radiation, so, the minimum gap between two photons for this two levels system is

$$
t_{g}=1 / A+1 / B \text {. }
$$

From the discussion in paragraph 1 and literature [4], we know $1 / A=T$ and $A / B=\frac{8 \pi \hbar \omega^{3}}{c^{3}}$, we obtain,

$$
t_{g}=T+\frac{8 \pi \hbar \omega^{3}}{c^{3}} T=\left(1+\frac{8 \pi \hbar \omega^{3}}{c^{3}}\right) T .
$$

Equation (12) tells us that $t_{g}$ is proportional to $\omega$, that is, the higher the frequency, the larger the $t_{g}$.

Now, we understand that the discontinuity of the light/electromagnetic wave makes the light/electromagnetic wave like a series of pulse or energy corpuscle. If the time resolution is close or beyond the $T$, we will find the interaction between photon and matter will last a certain time (period of light/electromagnetic wave), not like a real particle in ideal elastic collision. If the space resolution is beyond the wave length of light/electromagnetic wave, then we will see the light/electromagnetic wave more like a wave instead of particle. This is the real reason why sometime the light/electromagnetic wave behaviors like a particle, sometime, the light/electromagnetic wave becomes more like a wave. Therefore, we call the property of the light/electromagnetic wave as the particle-wave duality.
Based on the result above, we speculate how to modulate the photon individually. If we can modulate the photon individually, then it will find great application in many fields, such as communication, electronics, etc.

Generally, the Maxwell's equation [8] is a continuous equation, but now, we demonstrate the light/electromagnetic wave is not continuous at all. That is the reason why the Maxwell's equation cannot describe the particle behavior of light/electromagnetic wave. Then, what is the correct expression of the Maxwell's equation or how to make the Maxwell' equation still valid at the micro range? If the light/electromagnetic wave is not continuous, then how about the electric field and magnetic fields (even including gravity fields)? My understanding is that if the light/electromagnetic wave is discontinuous, then there is no reason for the electric fields and magnetic fields (even including gravity field) to be continuous. The electric fields and magnetic fields (even including gravity fields) must be discontinuous. The meaning for the discontinuity of the electric fields and magnetic fields (even including the gravity fields), at moment, is still unclear. Maybe it is the origin of the quantized electric fields and magnetic fields (even gravity fields). May be it is the discontinuity of the light/electromagnetic wave that is the basis of the quantized electric fields and magnetic fields (even gravity fields). We will keep working on these topics and present the result later.

\section{Summary}

In this paper we determined the spacetime dimension of a photon and the time for a system to absorb a photon is the period of light wave. This result reveals that the natural line width of the absorption and emission spectra is $2 T$. Based on the mechanism of the emission/absorp- 
tion of light in a system, we found that the light/electromagnetic wave is not continuous but discontinuous instead. This discontinuity of light/electromagnetic wave elucidates the origin of the duality more reasonably than before.

\section{REFERENCES}

[1] Y. Thomas, "Bakerian Lecture: Experiments and Calculations Relative to Physical Optics," Philosophical Transactions of the Royal Society of London, Vol. 94, 1804, pp. $1-16$.

[2] A. Einstein, "Zur Theorie der Radiometerkrafte," Zeitschrift Fuer Physik, Vol. 27, No. 1, 1924, pp. pp. 1-6.

[3] A. Einstein, "Uebereinen die Erzeugung und Verwandlung des Lichtes Betreffenden Heuristischen Gesichtspunkt," Annalen der Physik, Vol. 17, No. 6, 1905, pp. 132-148. doi:10.1002/andp.19053220607
[4] R. L. Liboff, "Introductory Quantum Mechinacs," Addison Wesley, San Francisco, 2003.

[5] A. Y. Davydov, "Wave-Particle Duality in Classical Mechanics," Journal of Physics: Conference Series, Vol. 361, No. 1, 2012, Article ID: 012029.

[6] M. Drescher and F. Krausz, "Attosecond Physics: Facing the Wave-Particle Duality," Journal of Physics B: Atomic, Molecular and Optical Physics, Vol. 38, No. 9, 2005, pp. s727-s740.

[7] H.-Y. Liu, J.-H. Huang, J.-R. Gao, Z. M. Suhail and S.-Y. Zhu, "Relation between Wave-Particle Duality and Quantum Uncertainty," Physical Review A, Vol. 85, No. 2, 2012, Article ID: 022106. doi:10.1103/PhysRevA.85.022106

[8] M. J. Clerk, "A Dynamical Theory of the Electromagnetic Field," Philosophical Transactions of the Royal Society of London, Vol. 155, 1865, pp. 459-512. doi:10.1098/rstl.1865.0008 